\title{
Serological response to influenza vaccine after hematopoetic stem cell transplantation
}

\author{
S. Songül Yalçın • Meda Kondolot • Nurhan Albayrak • A. Başak Altaș • \\ Yasemin Karacan • Barıș Kușkonmaz • Salih Aksu • Mualla Çetin • Hakan Göker • \\ Kadriye Yurdakök • Duygu Uçkan
}

Received: 9 November 2009/Accepted: 27 December 2009/Published online: 30 January 2010

(C) Springer-Verlag 2010

\begin{abstract}
Vaccination is the best strategy to prevent influenza infection that is a potential cause of morbidity and mortality in immunosuppressed patients. Here, we evaluated the factors that may affect serological response to influenza vaccine in patients who have undergone hematopoetic stem cell transplantation (HSCT). Sixty-one HSCT recipients were included in the study during the 2007-2008 influenza season. Serum samples prior to vaccination and 6-10 weeks after vaccination were collected. Samples were assayed for antibodies to influenza virus $\mathrm{A} / \mathrm{H} 1 \mathrm{~N} 1, \mathrm{~A} / \mathrm{H} 3 \mathrm{~N} 2$, and $\mathrm{B}$ strains by hemagglutination-inhibition assay. The patients were followed in terms of clinical symptoms up to the next influenza season and for adverse effects within a month after vaccination. Overall, pre-vaccine seroprotection rate against all vaccine antigens $(\mathrm{A} / \mathrm{H} 1 \mathrm{~N} 1, \mathrm{~A} / \mathrm{H} 3 \mathrm{~N} 2$, and $\mathrm{B}$ antigens) was $45.1 \%$, post-vaccine seroprotection rate $91 \%$ and seroconversion rate was $28.3 \%$. Seroconver-
\end{abstract}

S. S. Yalçın $(\bowtie) \cdot$ M. Kondolot $\cdot$ K. Yurdakök

Unit of Social Pediatrics, Department of Pediatrics,

Faculty of Medicine, Hacettepe University,

06100 Ankara, Turkey

e-mail: siyalcin@hacettepe.edu.tr

N. Albayrak $\cdot$ A. B. Altaș

Virology Unit of Refik Saydam Hifzıssiha Center,

Ankara, Turkey

Y. Karacan · S. Aksu $\cdot$ H. Göker

Unit of Hematopoetic Stem Cell Transplantation,

Department of Internal Medicine, Faculty of Medicine,

Hacettepe University,

Ankara, Turkey

B. Kuşkonmaz • M. Çetin • D. Uçkan

Unit of Hematopoetic Stem Cell Transplantation,

Department of Pediatrics, Faculty of Medicine,

Hacettepe University,

Ankara, Turkey sion rates were found to be low against $\mathrm{B}$ in patients who were vaccinated in the late influenza season $(p=0.018$; respectively). Five patients $(10.9 \%)$ had no immune response against H1N1. Adverse events were reported in $19.6 \%(n=9 / 46)$ of the patients. In conclusion, the patients should be vaccinated as early as possible in the influenza season, before they are exposed to the virus.

Keywords Influenza $\cdot$ Hematopoetic stem cell transplantation $\cdot$ Immunization $\cdot$ Seroconversion . Seroprotectivity $\cdot$ Adverse events

\section{Introduction}

Influenza epidemics have been the most important worldwide medical threats recently. Although influenza is a common cause of respiratory tract infection, it can be lifethreatening especially in immunosuppressed persons. The incidence of complications is particularly high in patients who had hematopoetic stem cell transplantation (HSCT), pneumonia being the leading one [1-9]. Influenzaassociated mortality has been reported to be $15 \%$ in HSCT recipients [1]. The incidence of influenza pneumonia varies from $9.5 \%$ to $75 \%$ in different studies $[1,6-8]$. In a bone marrow transplantation (BMT) unit, influenza A was detected in $23 \%$ and influenza $B$ in $15 \%$ of patients with symptoms of upper respiratory infections [4]. Nichols et al. reported that influenza pneumonia developed in $29 \%$ of the HSCT recipients who were infected with influenza virus and $28 \%$ of those patients died within 30 days [5]. Besides isolation precautions, vaccination is the main prophylactic approach in HSCT recipients for severe influenza disease or post-influenza complications [2, 3, 10-15]. However, the efficacy of influenza vaccine depends primarily on the age 
and immunocompetence of HSCT recipients and the degree of similarity between the viruses in the vaccine and those in circulation $[3,10]$. Both humoral immune response and the cell-mediated responses contribute to a successful protection against influenza $[2,16]$. In the present study, we aimed to evaluate the factors (gender, age at the time of the transplantation, time after HSCT, conditioning regimen, type of HSCT, history or presence of graft versus host disease (GVHD), underlying diseases, dose of the influenza vaccine, time of the influenza vaccination, past season influenza vaccination, smoke exposure, and donor age) that may affect serological response to influenza vaccination in HSCT recipients. Therefore, the aim was to determine the appropriate conditions to obtain the best response to the vaccination.

\section{Materials and methods}

Patients

The study was conducted during the 2007-2008 influenza season at Hacettepe University Institute of Child Health Department of Social Pediatrics (Ankara, Turkey). Patients were only excluded if there was a recognized contraindication to receipt of a vaccine. Clinical data on each patient were obtained from the patient, parents, and patient records. Informed consent was obtained from the patient or his/her parent. This study was approved by the Ethical Committee for Medical, Surgical, and Drug Research at Hacettepe University Faculty of Medicine, Ankara, Turkey.

Sixty-one HSCT recipients whose ages between 2 and 54 years (median 14 years) were included in the study during the 2007-2008 influenza season. Patients' characteristics are shown in Table 1. The patients were immunized from September 2007 to February 2008 with commercially available inactivated trivalent 2007/2008 influenza vaccines containing $15 \mathrm{mcg}$ hemagglutinin from each of the following three strains: A/Solomon Islands/3/2006 (H1N1), A/Wisconsin/67/ 2005 (H3N2), B/Malaysia/2506/2004 (B) [17].

Children younger than 9 years of age who have not previously been immunized against influenza were immunized two doses at least 1 month apart. Children older than 9 years of age and adults were immunized with one dose. The patients were followed for the presence of clinical symptoms up to next influenza season and for adverse events within 1 month following vaccination. To access risk factors, files of patients were reviewed retrospectively. Variables evaluated included gender, age at time of vaccination, duration after HSCT, conditioning regimen, type of HSCT, history or presence of graft versus host disease, underlying diseases, dose of the influenza vaccine, date of the vaccination, past season influenza vaccination,
Table 1 Patients' characteristics $(n=61)$

\begin{tabular}{ll}
\hline Characteristics & \\
\hline Age, median (range) & 14 years $(2-54)$ \\
Age at the time of the & 11 years $(2$ months-52 years) \\
transplantation, median (range) & $41(67.2)$ \\
Male, $n$ (\%) & \\
Underlying diseases, $n(\%)$ & $14(23)$ \\
Acute myeloid leukemia & $10(16.4)$ \\
Aplastic anemia & $6(9.8)$ \\
Acute lymphoblastic leukemia & $6(9.8)$ \\
Chronic myeloid leukemia & $6(9.8)$ \\
Thalassemia major & $6(9.8)$ \\
Fanconi aplastic anemia & $2(3.3)$ \\
Multiple myeloma & $2(3.3)$ \\
Osteopetrosis & $2(3.3)$ \\
Myelodysplastic syndrome & $7(11.4)$ \\
Others & \\
Type of HSCT, $n$ (\%) & $58(95.1)$ \\
Allogeneic & $3(4.9)$ \\
Autologous & $32(52.5)$ \\
Conditioning regimen, $n(\%)$ & $29(47.5)$ \\
Myeloablative & $19(31.1)$ \\
Reduced intensity & $19.2(6$ months-55 years) \\
Past season influenza & \\
vaccination, $n(\%)$ & \\
\hline
\end{tabular}

smoke exposure, and donor age. The patients were asked for any adverse events of influenza vaccine and symptoms of influenza infection by phone calls. They were also checked at every clinical visit. Influenza infection was diagnosed by clinical symptoms. Influenza-like illness was defined as individuals with fever $>37.5^{\circ} \mathrm{C}$ and at least one constitutional symptom and one respiratory symptom.

Immunogenecity assay-HAI assay

The pre-vaccination sera were collected prior to the vaccination, and the post-vaccination sera were collected at 6-10 weeks after vaccination. All sera were stored at $-20^{\circ} \mathrm{C}$ until they were tested. Samples were assayed for antibodies to influenza virus $\mathrm{A} / \mathrm{H} 1 \mathrm{~N} 1, \mathrm{~A} / \mathrm{H} 3 \mathrm{~N} 2$, and $\mathrm{B}$ strains. Hemagglutination inhibition (HAI) assay was performed in Refik Saydam Hygiene Center Viroloji Laboratory. Initially, serum non-specific inhibitors were treated with receptor destroying enzyme overnight at $37^{\circ} \mathrm{C}$, followed by inactivation at $56^{\circ} \mathrm{C}$ for $30 \mathrm{~min}$. Then, hemagglutination test was performed to detect the titer of each antigen, then standard antigen was diluted to contain four hemagglutinin units and back titration was realized. Samples were assayed for antibodies to influenza virus $\mathrm{A} / \mathrm{H} 1 \mathrm{~N} 1, \mathrm{~A} / \mathrm{H} 3 \mathrm{~N} 2$, and $\mathrm{B}$ 
strains by HAI assay using reference antiserum kindly supplied by World Health Organization for the influenza season 2007-2008. HAI antibody titers were determined with all of the paired specimens run in the same test. Sera were diluted 1:10, subsequently serial twofold dilutions were done. Twenty-five microliters of the diluted sera were incubated with an equal volume of antigen. Fifty microliters of a $0.5 \%$ suspension of human type ' 0 ' red blood cells was then added to the mixture. The HAI assay presented the highest reciprocal dilution which induced complete hemagglutination inhibition. A potentially protective antibody titer (seroprotection) was defined as an HAI titer 1/40. Seroconversion or significant titer increase was defined as a fourfold increase in the antibody titer after vaccination.

\section{Statistical analysis}

All analyses performed with SPSS for Windows (SPSS Inc., Chicago, IL, USA). The difference of case distribution between the groups was analyzed by using Chi-square test. $P$ values $<0.05$ were considered significant. Multiple logistic regression analysis was used to determine which factors among gender, age at the time of the HSCT ( $<10$ vs $10-17$ and $\geq 18$ years of age), duration after transplantation ( $<12$ vs $12-24$ and $\geq 24$ months), underlying diseases (malignant vs nonmalignant), conditioning regimen (myeloablative vs reduced intensity), type of HSCT (allogeneic vs autologous), vaccination dose (one, two), time of influenza vaccination season (September-October vs later), and prevaccine seropositivity best predicted seroconversion against all vaccine antigens.

\section{Results}

Patients' characteristics are shown in Table 1. Acute GVHD occurred in two patients. Prior to vaccination, 51 cases permitted to give blood samples, seroprotection rate was $47.1 \%$ for $\mathrm{H} 1 \mathrm{~N} 1,96.1 \%$ for $\mathrm{H} 3 \mathrm{~N} 2,92.2 \%$ for $\mathrm{B}$, and $45.1 \%(23 / 51)$ all three antigens $(\mathrm{A} / \mathrm{H} 1 \mathrm{~N} 1, \mathrm{~A} / \mathrm{H} 3 \mathrm{~N} 2$, and influenza B) of influenza virus. The following factors did not affect pre-vaccine seroprotection rates: gender, age at the time of vaccination, underlying disease, number of people in the house, presence of children in the house, previous influenza vaccination, and whether there are vaccinated people in the house (Table 2).

Post-vaccination 56 cases gave blood samples and postvaccine seroprotection rates were $91.0 \%$ against to $H 1 N 1$, $100.0 \%$ against to $\mathrm{H} 3 \mathrm{~N} 2$, and $100.0 \%$ against to $\mathrm{B} 1-2$ months after vaccination.

Seroconversion rates were $60.9 \%, 56.5 \%$, and $47.8 \%$ against $\mathrm{H} 1 \mathrm{~N} 1, \mathrm{H} 3 \mathrm{~N} 2$, and $\mathrm{B}$, respectively, and overall
(A/H1N1, A/H3N2, and B) seroconversion rate was $28.3 \%$ in cases vaccinated 6-48 months after HSCT. Vaccination age, duration after HSCT, underlying diseases, conditioning regimen, vaccination dose, pre-vaccine antibody titer, and smoking exposure did not affect seroconversion rates against three antigens of vaccine in cases vaccinated 6 months after HSCT. However, seroconversion rates were found to be low for B strain in cases vaccinated in the late influenza season $(\mathrm{OR}=0.14$, 95\%CI 0.026-0.740, $p=0.018$, Table 3). After adjustment for vaccination age, duration after HSCT ( $<12$ vs $\geq 12$ months), underlying diseases, conditioning regimen, vaccination dose, time of influenza vaccination season, and pre-vaccine antibody titer, cases vaccinated in the late influenza season had lower seroconversion rates for B strain $(\mathrm{OR}=0.102 ; 95 \% \mathrm{CI}, 0.013-0.820)$. Multivariate analysis showed that the frequency of seroconverted cases against all the vaccine antigens were also lower in cases vaccinated later in influenza season $(\mathrm{OR}=0.054$; 95\%CI, 0.003-0.886). Pre-vaccine seropositivity did not change seroconversion rates (Table 4).

Seroconversion rates for both $\mathrm{H} 1 \mathrm{~N} 1$ and $\mathrm{H} 3 \mathrm{~N} 2$ strains were $100 \%$ in two patients who received autologous transplant. After vaccination, 10.9\% (5/46) of patients had no immune response against to $\mathrm{H} 1 \mathrm{~N} 1$. All patients were seroresponsive to $\mathrm{H} 3 \mathrm{~N} 2$ and B strains.

Adverse events were observed in nine patients after influenza vaccination. Local side-effects (pain in five, pain and swelling in two, swelling, and erythema in one in the injection side) were observed in eight patients. One patient had systemic side-effects (flu-like symptoms).

The presence of influenza-like infection within 5 months following vaccination was similar in both seroconverted and non-seroconverted cases (Table 5). Two patients died at +27 months after transplantation, 10-11 months after vaccination due to relapse of underlying diseases.

\section{Discussion}

The long-lasting immunosuppressive period after HSCT constitutes a great risk of infection and also decreases the effectiveness of vaccinations. In the present study, $96 \%$ of the patients had seroprotective antibody levels against influenza $\mathrm{A} / \mathrm{H} 3 \mathrm{~N} 2,92 \%$ to influenza $\mathrm{B}$, and $47 \%$ to influenza $\mathrm{A} / \mathrm{H} 1 \mathrm{~N} 1$ prior to vaccination. Pauksen et al. [18] reported that the frequency of protective HAI antibodies was $12-16 \%$ prior to vaccination. In the present study, high seroprotection rates before vaccination showed that our patients were exposed more to influenza viruses prior to vaccination. This high seroprotection rates were unrelated to gender, age at the time of vaccination, underlying disease, number of people in the house, presence of children in the house, previous influenza 
Table 2 Seroprotection rates prior to influenza vaccination, $n(\%)^{*}$

\begin{tabular}{|c|c|c|c|c|c|c|}
\hline & & $N$ & H1N1 & H3N2 & $\mathrm{B}$ & All \\
\hline Overall, $n(\%)$ & & 51 & $24(47.0)$ & $49(96.0)$ & $47(92.0)$ & $23(45.1)$ \\
\hline \multirow[t]{2}{*}{ Gender, $n(\%)$} & Male & 34 & $18(52.9)$ & $33(97.1)$ & $33(97.1)$ & $18(52.9)$ \\
\hline & Female & 17 & $6(35.3)$ & $16(94.1)$ & $14(82.4)$ & $5(29.4)$ \\
\hline \multirow[t]{3}{*}{ Vaccination time after HSCT } & $6-11$ months & 4 & $2(50.0)$ & $3(75.0)$ & $3(75.0)$ & $2(50.0)$ \\
\hline & $12-23$ & 21 & $9(42.9)$ & $21(100.0)$ & $19(90.5)$ & $9(42.9)$ \\
\hline & $\geq 24$ & 26 & $13(50.0)$ & $25(96.2)$ & $25(96.2)$ & $12(46.2)$ \\
\hline \multirow[t]{3}{*}{ Age at the time of vaccination } & $\leq 9$ years & 17 & $6(35.3)$ & $17(100.0)$ & $16(94.1)$ & $6(35.3)$ \\
\hline & $10-17$ years & 14 & $6(42.9)$ & $13(92.9)$ & $13(92.9)$ & $6(42.9)$ \\
\hline & $\geq 18$ years & 20 & $12(60.0)$ & $19(95.0)$ & $18(90.0)$ & $11(55.0)$ \\
\hline \multirow[t]{2}{*}{ Underlying diseases } & Malignant & 22 & $11(50.0)$ & $21(95.5)$ & $19(86.4)$ & $10(45.5)$ \\
\hline & Nonmalignant & 29 & $13(44.8)$ & $28(96.6)$ & $28(96.6)$ & $13(44.8)$ \\
\hline \multirow[t]{2}{*}{ House contacts } & $<4$ persons & 25 & $13(52.0)$ & $24(96.0)$ & $22(88.0)$ & $12(48.0)$ \\
\hline & $\geq 4$ persons & 22 & $10(45.5)$ & $21(95.5)$ & $21(95.5)$ & $10(45.5)$ \\
\hline \multirow[t]{2}{*}{ Child at home } & 0 & 9 & $6(66.7)$ & $8(88.9)$ & $7(77.8)$ & $5(55.6)$ \\
\hline & $\geq 1$ & 38 & $17(44.7)$ & $37(97.4)$ & $36(94.7)$ & $17(44.7)$ \\
\hline \multirow[t]{2}{*}{ Past season influenza vaccination } & Yes & 14 & $8(57.1)$ & $13(92.9)$ & $13(92.9)$ & $7(50.0)$ \\
\hline & No & 36 & $16(44.4)$ & $35(97.2)$ & $33(91.7)$ & $16(44.4)$ \\
\hline \multirow[t]{2}{*}{ Time of influenza vaccination } & Sept-Oct & 36 & $15(41.7)$ & $34(94.4)$ & $32(88.9)$ & $14(38.9)$ \\
\hline & Nov + & 15 & $9(60.0)$ & $15(100.0)$ & $15(100.0)$ & $9(60.0)$ \\
\hline \multirow[t]{2}{*}{ Vaccinated person at home } & Yes & 17 & $7(41.2)$ & $16(94.1)$ & $16(94.1)$ & $7(41.2)$ \\
\hline & No & 30 & $16(53.3)$ & $29(96.7)$ & $27(90.0)$ & $15(50.0)$ \\
\hline
\end{tabular}

$* p>0.05$

vaccination, and whether there are vaccinated people in the house; however, we have no reported data about the frequency of health center admissions and we were unable to detect if hospital admission rates would make any difference. Also, specific antibody production can be detected after 3 months from HSCT because patients are more exposed to infections by circulating respiratory viruses [3]. Based on these findings, Avetisyan et al. [16] recommended influenza vaccination to the performed at later than 3 months after BMT, as long as they do not have GVHD or ongoing immunosupression.

Seroconversion rates in cases vaccinated 6-48 months after HSCT in the present study were detected similar to healthy controls and higher than HSCT cases $(60.9 \%$, $56.5 \%$, and $47.8 \%$ against $\mathrm{H} 1 \mathrm{~N} 1, \mathrm{H} 3 \mathrm{~N} 2$, and $\mathrm{B}$, respectively). In this study, we have not a healthy control group but Pauksen et al. have reported seroconversion rates of $46 \%$ against $\mathrm{H} 1 \mathrm{~N} 1,54 \%$ against $\mathrm{H} 3 \mathrm{~N} 2$, and $62 \%$ against $\mathrm{B}$ in healthy control subjects [18]. Avetisyan et al. [16] demonstrated that $29 \%$ of the patients had protective antibody levels to $\mathrm{H} 1 \mathrm{~N} 1$, whereas none of them were immune to $\mathrm{H} 3 \mathrm{~N} 2$ and $\mathrm{B}$ in cases vaccinated 3-24 months after BMT. Pauksen et al. [18] reported that 4-24 months after BMT $(n=117)$, antibody response to $\mathrm{H} 1 \mathrm{~N} 1$ after vaccination was $29 \%$, response to $\mathrm{H} 3 \mathrm{~N} 3$ was $25 \%$, and to $\mathrm{B}$ was $34 \%$. One plausible explanation is that cases were vaccinated later in the present study, 6-48 months after HSCT than the other studies. Bone marrow transplant recipients may suffer from immunological deficiency up to several years after transplant. In the absence of chronic GVHD, normalization of B-cell function is considered to take place within 1 to 2 years. Although B-cell numbers become normal at 4 to 8 months post-transplant, their phenotype is immature and their function is inadequate [19-21]. Engelhard et al. [21] showed that vaccination of BMT recipients was ineffective if the immunization was performed earlier than 6 months after BMT.

Following vaccination, $10.9 \%(5 / 46)$ of the patients did not have any immune response to H1N1. It is interesting that these five seronegative patients had received myeloablative regimens and were all children. Interestingly, three cases of them showed seroconversion to $\mathrm{H} 3 \mathrm{~N} 2$ and only one case to $\mathrm{B}$. It is reported that reduced intensity conditioning regimens have been associated with a better short-term immune reconstitution than conventional regimens, but with no difference at long-term [22]. Morecki et al. [19] concluded that stem cell engraftment following reduced intensity conditioning may result in early reconstitution of immune responses assessed in vitro. Therefore, it is important to decrease the exposure to influenza by vaccinating family contacts and hospital staff early after HSCT. 
Table 3 The factors that may affect seroconversion rates against influenza vaccination, $n(\%)$

\begin{tabular}{|c|c|c|c|c|c|c|}
\hline & & $n$ & H1N1 & $\mathrm{H} 3 \mathrm{~N} 2$ & $\mathrm{~B}$ & All \\
\hline Overall, $n(\%)$ & & 46 & $28(60.9)$ & $26(56.5)$ & $22(47.8)$ & $13(28.3)$ \\
\hline \multirow[t]{2}{*}{ Sex, $n(\%)$} & Male & 30 & $19(63.3)$ & $17(56.7)$ & $15(50.0)$ & $9(30.0)$ \\
\hline & Female & 16 & $9(56.2)$ & $9(56.2)$ & $7(43.8)$ & $4(25.0)$ \\
\hline \multirow[t]{3}{*}{ Age at the time of vaccination } & $\leq 9$ years & 14 & $10(71.4)$ & $10(71.4)$ & $6(42.9)$ & $6(42.9)$ \\
\hline & $10-17$ & 13 & $5(38.5)$ & $6(46.2)$ & $5(38.5)$ & $2(15.4)$ \\
\hline & $\geq 18$ & 19 & $13(68.4)$ & $10(52.6)$ & $11(57.9)$ & $5(26.3)$ \\
\hline \multirow[t]{3}{*}{ Vaccination time after HSCT } & 6-11 months & 4 & $2(50.0)$ & $1(25.0)$ & $1(25.0)$ & $1(25.0)$ \\
\hline & $12-23$ & 20 & $11(55.0)$ & $12(60.0)$ & $9(45.0)$ & $6(30.0)$ \\
\hline & $\geq 24$ & 22 & $15(68.2)$ & $13(59.1)$ & $12(54.5)$ & $6(27.3)$ \\
\hline \multirow[t]{2}{*}{ Conditioning regimen } & Myeloablative & 23 & $13(56.5)$ & $14(60.9)$ & $9(39.1)$ & $6(26.1)$ \\
\hline & Reduced intensity & 23 & $15(65.2)$ & $12(52.2)$ & $13(56.5)$ & $7(30.4)$ \\
\hline \multirow[t]{2}{*}{ Type of HSCT } & Allogeneic & 44 & $26(59.1)$ & $24(54.5)$ & $21(47.7)$ & $12(27.3)$ \\
\hline & Autologous & 2 & $2(100.0)$ & $2(100.0)$ & $1(50.0)$ & $1(50.0)$ \\
\hline \multirow[t]{2}{*}{ Underlying disease } & Malignant & 20 & $13(65.0)$ & $12(60.0)$ & $9(45.0)$ & $5(25.0)$ \\
\hline & Nonmalignant & 26 & $15(57.7)$ & $14(53.8)$ & $13(50.0)$ & $8(30.8)$ \\
\hline \multirow[t]{2}{*}{ Vaccination dose } & One & 36 & $20(55.6)$ & $18(50.0)$ & $17(47.2)$ & $8(22.2)$ \\
\hline & Two & 10 & $8(80.0)$ & $8(80.0)$ & $5(50.0)$ & $5(50.0)$ \\
\hline \multirow[t]{2}{*}{ Time of influenza vaccination } & Sept-Oct & 34 & $21(61.8)$ & $21(61.8)$ & $20(58.8)^{*}$ & $12(35.3)$ \\
\hline & Nov + & 12 & $7(58.3)$ & $5(41.7)$ & $2(16.7)$ & $1(8.3)$ \\
\hline \multirow[t]{2}{*}{ Past season influenza vaccination } & Yes & 14 & $9(64.3)$ & $8(57.1)$ & $7(50.0)$ & $3(21.4)$ \\
\hline & No & 32 & $19(59.4)$ & $18(56.2)$ & $15(46.9)$ & $10(31.3)$ \\
\hline \multirow[t]{2}{*}{ Smoke exposure } & Yes & 17 & $8(47.1)$ & $11(64.7)$ & 7 (41.2) & $4(23.5)$ \\
\hline & No & 26 & $17(65.4)$ & $14(53.8)$ & $13(50.0)$ & $8(30.8)$ \\
\hline
\end{tabular}

$* p<0.05$

Table 4 Seroconversion against vaccine antigens according to selected antigens*

\begin{tabular}{llr}
\hline Vaccine antigens & \multicolumn{2}{c}{$\begin{array}{l}\text { Seroconversion against } \\
\text { vaccine antigens }\end{array}$} \\
\cline { 2 - 3 } & $n$ & $\%$ \\
\hline H1N1 & & 47.6 \\
Seropositive prior to vaccination & $10 / 21$ & 72.0 \\
Seronegative prior to vaccination & $18 / 25$ & \\
H3N2 & & 54.5 \\
Seropositive prior to vaccination & $24 / 44$ & 100.0 \\
Seronegative prior to vaccination & $2 / 2$ & 47.6 \\
B & & 50.0 \\
Seropositive prior to vaccination & $20 / 42$ & 20.0 \\
Seronegative prior to vaccination & $2 / 4$ & 34.6 \\
All vaccine antigens & & \\
Seropositive prior to vaccination & $4 / 20$ & \\
Seronegative prior to vaccination & $9 / 26$ & \\
\hline
\end{tabular}

$* p>0.05$
Seroconversion rate for influenza B was low in patients vaccinated in the late influenza season. This significance was also detected after adjustment of confounding factors. It may be due to acquired influenza $B$. Therefore, the patients should be vaccinated as early as possible in the influenza season. Further studies are necessary to evaluate this subject.

Table 5 Reported influenza-like infection during 5 months after vaccination, $(n=43), n(\%)^{*}$

\begin{tabular}{lll}
\hline Vaccine antigens & $\begin{array}{l}\text { Seroconversion } \\
\text { against antigens }\end{array}$ & $\begin{array}{l}\text { Influenza-like } \\
\text { infection after } \\
\text { influenza vaccination }\end{array}$ \\
\hline H1N1 & Seroconverted & $2 / 25(8.0)$ \\
H3N2 & Non-seroconverted & $5 / 18(27.8)$ \\
& Seroconverted & $5 / 25(20.0)$ \\
B & Non-seroconverted & $2 / 18(11.1)$ \\
& Seroconverted & $4 / 20(20.0)$ \\
All vaccine antigens & Non-seroconverted & $3 / 23(13.0)$ \\
(H1N1+H3N2+B) & Neroconverted & $2 / 12(16.7)$ \\
\hline
\end{tabular}

${ }^{*} p>0.05$ 
Cases who seroconverted for vaccine strains had similar rates for having influenza-like illness within the 5 months following vaccination with non-seroconverted. However, Machado et al. [3] reported influenza infection among $10.5 \%$ of vaccinated patients $(2 / 19)$ and $50 \%$ of unvaccinated (12/24). Influenza vaccination efficiency was determined to be $80 \%$ in their study but the serological response was not evaluated. As a limitation of the present study, influenza infection was considered according to the presence of clinical symptoms without laboratory confirmation.

Seroconversion is also more likely to occur in autologous HSCT in comparison to allogeneic [3, 23]. In our study, antibody response to H1N1 and H3N2 was $100 \%$ in two autologous transplant patients but, statistical analysis was not performed due to the limited number of the patients. Also, Nichols et al. reported that donor type was not associated with the risk for influenza virus infection [5].

The reported side-effects of influenza vaccine have been mild and have not been different than healthy controls [2]. In our study, mild adverse events were observed after influenza vaccination $(19.6 \%(n=9 / 46))$ and $88.9 \%$ of them were local. Pauksen et al. [18] reported severe events in five patients (8\%) who received GM-CSF and in none for those who did not.

In conclusion, pre-vaccination seroprotection rates against $\mathrm{H} 3 \mathrm{~N} 2$ and $\mathrm{B}$ antigens were found to be high in our BMT recipients during the 2007/2008 influenza season. Seroconversion rates in cases vaccinated 6-48 months after HSCT in the present study were found to be similar to healthy cases. However, presence of influenza-like symptoms was also found to be similar in both seroconverted and non-seroconverted cases during 5 months of follow-up after immunization but this result can be related with our limited number of cases. It is important that the patients should be vaccinated as early as possible in the influenza season, before they are exposed to the virus.

\section{References}

1. Ljungman P, Ward KN, Crooks BN, Parker A, Martino R, Shaw PJ et al (2001) Respiratory virus infections after stem cell transplantation: a prospective study from the Infectious Diseases Working Party of the European Group for Blood and Marrow Transplantation. Bone Marrow Transplant 28:479-484

2. Ljungman P, Avetisyan G (2008) Influenza vaccination in hematopoietic SCT recipients. Bone Marrow Transplant 42:637-641

3. Machado CM, Cardoso MRA, da Rocha IF, Boas LSV, Dulley FL (2005) The benefit of influenza vaccination after bone marrow transplantation. Bone Marrow Transplant 36:897-900

4. Raboni SM, Nogueira MB, Tsuchiya LR, Takahashi GA, Pereira LA, Pasquini R, Siqueira MM (2003) Respiratory tract viral infections in bone marrow transplant patients. Transplantation 76:142-146

5. Nichols WG, Guthrie KA, Corey L, Boeckh M (2004) Influenza infections after hematopoietic stem cell transplantation: risk factors, mortality, and the effect of antiviral therapy. Clin Infect Dis 39:1300-1306
6. Whimbey E, Elting LS, Couch RB, Lo W, Williams L, Champlin RE, Bodey GP (1994) Influenza A virus infections among hospitalized adult bone marrow transplant recipients. Bone Marrow Transplant 13:437-440

7. Bowden RA (1997) Respiratory virus infections after marrow transplant: The Fred Hutchinson Cancer Research Center Experience. Am J Med 102:27-30

8. Cough RB, Englund JA, Whimbley E (1997) Respiratory viral infections in immunocompetent and immunocompromised persons. Am J Med 102:2-9

9. Scott JD, Englund JA, Myerson D, Geballe AP (2004) Influenza a pneumonia presenting as progressive focal infiltrates in a stem cell transplant recipient. J Clin Virol 31:96-99

10. Fiore AE, Shay DK, Broder K, Iskander JK, Uyeki TM, Mootrey $\mathrm{G}$ et al (2008) Centers for Disease Control and Prevention (CDC); Advisory Committee on Immunization Practices (ACIP). Prevention and control of influenza: recommendations of the Advisory Committee on Immunization Practices (ACIP) 2008. MMWR Recomm Rep 57:1-60

11. Van Essen GA, Palache AM, Forleo E, Fedson DS (2003) Influenza vaccination in 2000: recommendations and vaccine use in 50 developed and rapidly developing countries. Vaccine 21:1780-1785

12. Ljungman P, Engelhard D, de la Camara R, Einsele H, Locasciulli A, Martino R et al (2005) Vaccination of stem cell transplant recipients: recommendations of the Infectious Diseases Working Party of the EBMT. Bone Marrow Transplant 35:737-746

13. Machado CM (2004) Reimmunization after bone marrow transplantation-current recommendations and perspectives. Braz J Med Biol Res 37:151-158

14. Zimmerman RK, Middleton DB (2007) Vaccines for persons at high risk. J Fam Pract 56(2):S38-S46

15. Wilck MB, Baden LR (2008) Vaccination after stem cell transplant: a review of recent developments and implications for current practice. Curr Opin Infect Dis 21:399-408

16. Avetisyan G, Aschan J, Hassan M, Ljungman P (2008) Evaluation of immune responses to seasonal influenza vaccination in healthy volunteers and in patients after stem cell transplantation. Transplantation 86:257-263

17. www.cdc.gov/flu/weekly/fluactivity.htm

18. Pauksen K, Linde A, Hammarström V, Sjölin J, Carneskog J, Jonsson G, Oberga G, Engelmann H, Ljungman P (2000) Granulocyte-macrophage colony-stimulating factor as immunomodulating factor together with influenza vaccination in stem cell transplant patients. Clin Infect Dis 30:342-348, Erratum in: Clin Infect Dis 2000; 30: 992

19. Morecki S, Gelfand Y, Nagler A, Or R, Naparstek E, Varadi G, Engelhard D, Akerstein A, Slavin S (2001) Immune reconstitution following allogeneic stem cell transplantation in recipients conditioned by low intensity vs myeloablative regimen. Bone Marrow Transplant 28:243-249

20. Ring A, Marx G, Steer C, Harper P (2002) Influenza vaccination and chemotherapy: a shot in the dark? Support Care Cancer 10:462-465

21. Engelhard D, Nagler A, Hardan I, Morag A, Aker M, Baciu H et al (1993) Antibody response to a two-dose regimen of influenza vaccine in allogeneic $\mathrm{T}$ cell depleted and autologous BMT recipients. Bone Marrow Transplant 11:1-5

22. Maris M, Boeckh M, Storer B, Dawson M, White K, Keng M, Sandmaier B, Maloney D, Storb R, Storek J (2003) Immunologic recovery after hematopoietic cell transplantation with nonmyeloablative conditioning. Exp Hematol 31:941-952

23. Gandhi MK, Egner W, Sizer L, Inman I, Zambon M, Craig JI, Marcus RE (2001) Antibody responses to vaccinations given within the first two years after transplant are similar between autologous peripheral blood stem cell and bone marrow transplant recipients. Bone Marrow Transplant 28:775-781 